\title{
The Effect of the Prognostic Nutritional Index on the Toxic Side Effects of Radiochemotherapy and Prognosis After Radical Surgery for Gastric Cancer
}

This article was published in the following Dove Press journal:

Cancer Management and Research

\author{
Ji-Yu Liu' \\ Hong-Min Dong ${ }^{2,3}$ \\ Wen-Ling Wang ${ }^{2,3}$ \\ Gang Wang ${ }^{1-3}$ \\ Huan $\operatorname{Pan}^{3}$ \\ Wei-Wei Chen ${ }^{1-3}$ \\ Qian Wang ${ }^{4}$ \\ Ze-Jun Wang ${ }^{5}$
}

'Department of Oncology, Guizhou Medical University, Guizhou, Guiyang, 55000 I, People's Republic of China; ${ }^{2}$ Department of Oncology, Affiliated Hospital of Guizhou Medical University, Guizhou, Guiyang, 55000I, People's Republic of China; ${ }^{3}$ Department of Abdominal Oncology, Guizhou Cancer Hospital, Guizhou, Guiyang, 55000I, People's Republic of China; ${ }^{4}$ Department of Gastrointestinal Surgery, Affiliated Hospital of Guizhou Medical University, Guizhou, Guiyang, 55000I, People's Republic of China; ${ }^{5}$ Department of Gastrointestinal Surgery, Guizhou Cancer Hospital, Guizhou, Guiyang, 55000I, People's Republic of China
Correspondence: Hong-Min Dong Department of Abdominal Oncology, Guizhou Cancer Hospital, NO. I Of West Beijing Road, Yunyan District, Guizhou, Guiyang, 55000I, People's Republic of China

Tel +86 85I-6855II9

Email donghm903hm@163.com
Objective: A retrospective analysis was conducted to investigate the effect of the preoperative prognostic nutritional index (PNI) on the severity of toxic side effects of radiochemotherapy and the survival prognosis of patients with gastric cancer to guide the clinical nutritional support for patients with gastric cancer.

Methods: Data of 191 patients with gastric cancer in the Department of Gastrointestinal Surgery of Guizhou Cancer Hospital and the Affiliated Hospital of Guizhou Medical University between January 2008 and December 2018 were analyzed retrospectively. Patients were allocated to the high PNI group (with PNI $\geq 47.7$ ) and the low PNI group (with PNI <47.7) according to the PNI cutoff value, and the incidence of severe toxic side effects of radiochemotherapy and the overall survival time were compared between the high PNI group and low PNI group. In addition, prognostic factor analysis was performed.

Results: The severe hematologic side effects of radiochemotherapy and shorter postoperative survival time were more likely to occur in the low PNI group than in the high PNI group. The multifactor analysis showed that TNM stage $(p=0.000)$ and PNI $(p=0.001)$ were the independent risk factors for the overall postoperative survival time in patients with gastric cancer. Conclusion: Preoperative PNI might predict the severity of hematologic toxic side effects of adjuvant chemotherapy/radiochemotherapy in patients with gastric cancer after surgery. Patients in the low PNI group were more likely to have severe hematologic toxic side effects, and therefore a low PNI might be one of the important factors affecting the prognosis of gastric cancer.

Keywords: stomach neoplasms, prognosis nutrition index, toxic side effects, prognosis

\section{Introduction}

Gastric cancer, malignant tumors of the digestive tract, is one of the most common cancer types in China. According to the relevant diagnostic and therapeutic standards of gastric cancer, ${ }^{1-3}$ patients with stage Ib gastric cancer can be treated with simple surgery or surgery combined with radiochemotherapy. Patients with locally advanced stage II/III gastric cancer can be treated with radical surgery and adjuvant chemotherapy or radiochemotherapy, and some patients can be treated with neoadjuvant therapy followed by surgery and adjuvant chemotherapy or radiochemotherapy. Systemic chemotherapy is usually adopted as the main treatment in patients with metastatic gastric cancer. Therefore, radiochemotherapy has become one of the 
important methods of treatment for patients with gastric cancer. The occurrence of severe side effects of radiochemotherapy can reduce the treatment compliance and effectiveness, leading to discontinuation or delay of treatment and reduced quality of life. Patients with gastric cancer are likely to have malnutrition due to anorexia, mechanical factors, and metabolic disorders, which affects their prognosis and survival. ${ }^{4}$ The prognostic nutritional index (PNI) was first defined by Onodera. It consists of the level of serum albumin and lymphocyte counts, which may reflect the nutritional and immune status in patients with cancer. ${ }^{5}$ A study on esophageal cancer shows that PNI is related to the severity of hematological toxicity of radiotherapy and chemotherapy. ${ }^{6}$ At present, there are few studies on the correlation between PNI and chemotherapy toxicity of gastric cancer, and no reports on the correlation between PNI and radiotherapy toxicity of gastric cancer. In the present study, the effect of the PNI on the degree of toxic side effects and the survival prognosis of adjuvant radiochemotherapy were investigated in patients with gastric cancer after surgery.

\section{Materials and Methods Collection of Clinical Data}

The data of patients with gastric cancer in the Department of Gastrointestinal Surgery of Guizhou Cancer Hospital and the Affiliated Hospital of Guizhou Medical University between January 2008 and December 2018 were collected retrospectively. The inclusion criteria were as follows: 1) patients with histopathologically confirmed gastric adenocarcinoma (including adenocarcinoma of the gastroesophageal junction, gastric body, and gastric sinus); 2) patients in postoperative pathologic stage $\mathrm{Ib}-\mathrm{IIIc}$ (the 8th edition American Joint Committee on Cancer (AJCC) TNM stage of gastric cancer) who underwent radical surgery (R0 resection) for gastric cancer; and 3) patients who received adjuvant chemotherapy or adjuvant chemotherapy combined with concomitant radiotherapy after the surgery. The exclusion criteria were as follows: 1) patients who had received parenteral nutrition therapy within 1 month before surgery; 2) patients who had perioperative blood transfusions; 3) patients who received preoperative neoadjuvant therapy; 4) patients with infectious diseases before the blood sample collection and patients with severe medical comorbidities before the surgery; 5) patients complicated with a second primary tumor; and 6) patients lost during the follow-up period. The data collected from the enrolled patients included gender, age, the results of the total peripheral blood lymphocyte count and serum albumin one week before the surgery, body mass index (BMI), degree of tumor differentiation, postoperative pathologic stage and postoperative adjuvant treatment regimen, results of the routine blood test together with the hepatic and renal function during the adjuvant radiochemotherapy, gastrointestinal tract reaction, skin mucosal reaction, and peripheral neuropathy. This study was approved by the ethics committee of Guizhou Medical University Hospital, and informed consent was obtained from all participants.

\section{Methods}

(1) Grouping: According to the results of the peripheral blood tests one week before the surgery, the preoperative PNI was calculated [formula: PNI = level of serum albu$\min (\mathrm{g} / \mathrm{L})+5 \times$ total number of peripheral blood lymphocytes $\left.\left(\times 10^{9} / \mathrm{L}\right)\right]$, and then the Receiver Operating Characteristic curve was plotted and the Youden index was calculated. With the PNI value corresponding to the highest Youden index being taken as the cutoff value, the patients were allocated to the preoperative high PNI group and the preoperative low PNI group.

(2) Evaluation of toxic side effects: The toxic side effects of radiochemotherapy were classified as 1, 2, 3, or 4 according to the National Cancer Institute Common Terminology Criteria for Adverse Events 3.0 criteria. ${ }^{7}$ The most severe hematologic or non-hematologic toxic side effects that occurred during the radiochemotherapy were used to evaluate the toxicity levels.

(3) Follow-up of survival: The survival time of all the enrolled patients was followed up through the outpatient review or telephone follow-up.

(4) Analysis of the relationship between PNI and mild and severe hematologic and non-hematologic toxic side effects of radiochemotherapy: The survival curves were plotted for the high and low PNI groups, and the clinicopathological factors (gender, age, differentiation, neurological or vascular infiltration, TNM stage, treatment mode, BMI, PNI, and cycles of chemotherapy) were included in the single factor and multifactor analyses of the survival prognosis.

\section{Statistical Analysis}

SPSS 25 software was used for the data analysis. The Kruskal-Wallis test and the independent $T$-test were used to analyze the relationship between PNI and the mild and severe 
toxic side effects of radiochemotherapy. The PNI values and 5-year survival time were adopted to plot the ROC curves. The best intercept of PNI was taken, and survival curves were plotted by using the Kaplan-Meier method. The Cox regression model was used for the single factor and multifactor analysis of the overall postoperative survival time. $\mathrm{P}<$ 0.05 was considered statistically significant.

\section{Results}

\section{General Characteristics of the Patients}

The data of 224 patients who received adjuvant chemotherapy and radiochemotherapy after undergoing radical surgery for gastric cancer were collected with the exclusion of 15 patients who received adjuvant chemotherapy with single-agent tigeo, 13 patients who received chemotherapy with a DP (Docetaxel + Oxaliplatin) regimen, and 5 patients who died of non-cancer -related diseases. Finally, 191 patients with gastric cancer were included in the present study. There were 128 males and 63 females aged 26-79 years (median age of 56 years). There were 10,47 , and 134 patients with stage Ib, stage II, and stage III gastric cancer, respectively, of which 156 patients had lowdifferentiated adenocarcinomas, 35 had intermediate- to highdifferentiated adenocarcinomas, and 79 had nerve or vascular invasion. All the patients underwent postoperative adjuvant chemotherapy (121 patients completed three-five therapeutic cycles, and 70 patients completed six-eight cycles). Adjuvant chemotherapy regimens included the XELOX regimen (oxaliplatin $130 \mathrm{mg} / \mathrm{m}^{2} \mathrm{~d} 1$, capecitabine $1000 \mathrm{mg} / \mathrm{m}^{2} \mathrm{~d} 1-\mathrm{d} 14$, and 21 days for one cycle) in 97 patients, and the SOX regimen (oxaliplatin $130 \mathrm{mg} / \mathrm{m}^{2} \mathrm{~d} 1$, tigeo $80 \mathrm{mg} / \mathrm{m}^{2} \mathrm{~d} 1-\mathrm{d} 14$, and 21 days for one cycle) in 94 cases. Among these, 93 cases were treated with adjuvant chemotherapy for one-two cycles followed by sequential concomitant radiochemotherapy. The regimen of the concomitant radiochemotherapy was as follows. The radiation area included the anastomosis + high-risk area of lymphatic drainage (T4b, radiotherapy area including the invasion area of the tumor bed). The total dosage was 45Gy/25f, 1.8Gy per time, 5-Fu225 mg/m2/day, and continuous intravenous pumped sensitization chemotherapy was given simultaneously. The characteristics of the enrolled patients are shown in Table 1.

\section{The ROC Curves}

The ROC curves were plotted according to the PNI values and 5-year survival time (Figure 1, the area under the curve was 0.706). When the PNI value was 47.77, the Youden index was the highest (78\% sensitivity, 53\%
Table I The Relationship Between the Clinicopathological Characteristics and PNI in Patients

\begin{tabular}{|c|c|c|c|c|}
\hline \multirow{2}{*}{$\begin{array}{l}\text { Clinicopathological } \\
\text { Characteristics }\end{array}$} & \multirow{2}{*}{$\begin{array}{l}\text { Total } \\
\text { Cases }\end{array}$} & \multicolumn{2}{|c|}{ PNI } & \multirow[t]{2}{*}{$\mathbf{P}$} \\
\hline & & $\begin{array}{l}\text { High PNI } \\
\text { Group } \\
\text { (I0I Cases) }\end{array}$ & $\begin{array}{l}\text { Low PNI } \\
\text { Group } \\
\text { (90 Cases) }\end{array}$ & \\
\hline \multicolumn{5}{|l|}{ Gender } \\
\hline Male & 128 & 65 & 63 & 0.408 \\
\hline Female & 63 & 36 & 27 & \\
\hline \multicolumn{5}{|l|}{ Age } \\
\hline$\geq 65$ years & 48 & 16 & 32 & 0.002 \\
\hline$<65$ years & 143 & 85 & 58 & \\
\hline \multicolumn{5}{|l|}{ BMI } \\
\hline$<18.5 \mathrm{~kg} / \mathrm{m} 2$ & 44 & 23 & 21 & 0.927 \\
\hline$\geq 18.5 \mathrm{~kg} / \mathrm{m} 2$ & 147 & 78 & 69 & \\
\hline \multicolumn{5}{|l|}{$\begin{array}{l}\text { Degree of differentiation } \\
\text { in tumor }\end{array}$} \\
\hline Low-differentiated & 156 & 82 & 74 & 0.854 \\
\hline $\begin{array}{l}\text { Intermediate to } \\
\text { high-differentiated }\end{array}$ & 35 & 19 & 16 & \\
\hline \multicolumn{5}{|l|}{$\begin{array}{l}\text { Nerve or vascular } \\
\text { invasion }\end{array}$} \\
\hline With invasion & 79 & 43 & 36 & 0.718 \\
\hline Without invasion & 112 & 58 & 54 & \\
\hline \multicolumn{5}{|l|}{ TNM Stage } \\
\hline Phase I-II & 57 & 35 & 22 & 0.124 \\
\hline Phase III & 134 & 66 & 68 & \\
\hline \multicolumn{5}{|l|}{ Radiotherapy } \\
\hline With radiotherapy & 93 & 47 & 46 & 0.528 \\
\hline Without radiotherapy & 98 & 54 & 44 & \\
\hline \multicolumn{5}{|l|}{ Cycles of chemotherapy } \\
\hline $3-5$ cycles & 121 & 62 & 59 & 0.551 \\
\hline 6-8 cycles & 70 & 39 & 31 & \\
\hline
\end{tabular}

Abbreviations: PNI, prognostic nutrition index; Low PNI group, PNI 47.77 ; High PNI group, PNI>47.77; BMI, body mass index;TNM, tumor-node-metastasis.

specificity). With 47.77 being taken as the cutoff value, the patients were allocated to the high PNI group (101 patients) and the low PNI group (90 patients), as shown in Table 1.

\section{The Toxic Side Effects of Radiochemotherapy}

Of the 191 patients with gastric cancer, all had completed three-eight cycles of adjuvant chemotherapy; in 93 patients, it had been combined with the concomitant radiochemotherapy. Hematologic side effects of the adjuvant chemotherapy above grade 3 occurred in 77 cases, as shown in Table 2. Hematologic side effects of the 


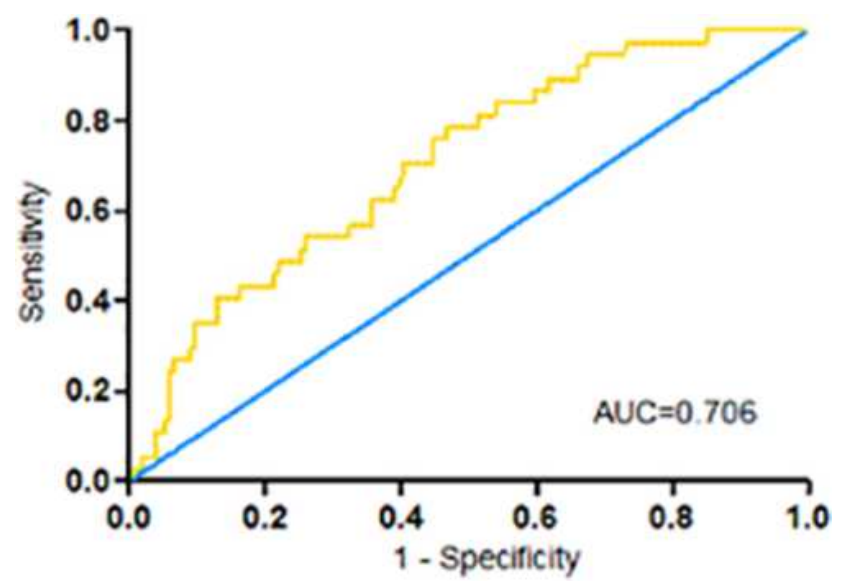

Figure I ROC curve of the prognostic nutritional index (AUC=0.706).

concomitant radiochemotherapy above grade 3 occurred in 26 cases, the details of which are provided in Table 3.

The mean PNI values in patients who experienced grade $0-2$ and grade 3-4 hematologic side effects of the adjuvant chemotherapy were $49.18 \pm 5.32$ and $45.73 \pm 6.02$, respectively, and the difference was statistically significant $(\mathrm{p}=0.000$, Figure $2 \mathrm{~A})$. The mean PNI values in patients who experienced grade $0-2$ and grade 3-4 hematologic side effects of the concomitant radiochemotherapy were $48.79 \pm 5.69$ and $45.52 \pm$ 6.99, respectively, and the difference was statistically significant $(p=0.030$, Figure $2 \mathrm{C})$. However, there was no significant difference in the mean PNI values in patients who experienced grade $0-2$ and grade 3-4 non-hematologic side effects of the adjuvant chemotherapy (the mean PNI value in those with grade $0-2$ was $48.00 \pm 5.91$, the mean PNI value in those with grade $3-4$ was $48.32 \pm 4.84, p=0.804$, Figure 2B). Furthermore, there was no significant difference in the mean PNI values in patients who experienced grade 0-2 and grade 3-4 non-hematologic side effects of the concomitant radiochemotherapy (the mean PNI values in those with grade $0-2$ was $48.13 \pm 6.22$, the mean PNI value in those with grade $3-4$ was $47.09 \pm 4.96$, $\mathrm{p}=0.667$, Figure 2D).

In the high PNI group, hematologic side effects of the adjuvant chemotherapy above grade 3 occurred in 21 cases (20.8\%), and hematologic side effects of the concomitant radiochemotherapy above grade 3 occurred in 7 cases (14.9\%). In the low PNI group, hematologic side effects of the adjuvant chemotherapy above grade 3 occurred in 42 patients $(46.7 \%)$, and hematologic side effects of the concomitant radiochemotherapy above grade 3 occurred in 14 patients (30.4\%).

\section{Prognosis of Survival}

The median duration of the follow-up was 58 months (4-130 months). The survival curve was plotted using the KaplanMeier method (Figure 3). The median survival time was 60 months and 32 months in the high PNI group and the low PNI group, respectively, The 1-, 2-, and 3-year survival rates were

Table 2 The Relationship Between the PNI and the Toxic Side-Effects of Adjuvant Chemotherapy Case (Rate)

\begin{tabular}{|c|c|c|c|c|c|c|}
\hline \multirow[t]{2}{*}{ Toxic Side-Effects of Chemotherapy } & \multicolumn{3}{|c|}{ High PNI Group (I0I Cases) } & \multicolumn{3}{|c|}{ Low PNI Group (90 Cases) } \\
\hline & Grade 0 & Grade I-2 & Grade 3-4 & Grade 0 & Grade I-2 & Grade 3-4 \\
\hline \multicolumn{7}{|l|}{ Hematologic toxic side effects } \\
\hline Leukocytosis & $39(38.6 \%)$ & $54(53.5 \%)$ & $8(7.9 \%)$ & $30(33.3 \%)$ & $38(42.2 \%)$ & $22(24.4 \%)$ \\
\hline Neutropenia & $39(38.6 \%)$ & $48(47.5 \%)$ & $14(13.9 \%)$ & $36(40.0 \%)$ & $28(31.1 \%)$ & $26(28.9 \%)$ \\
\hline Decrease in hemoglobin & $68(67.3 \%)$ & $30(29.7 \%)$ & $3(3.0 \%)$ & $45(50.0 \%)$ & $36(40.0 \%)$ & $9(10.0 \%)$ \\
\hline Decrease in hemoglobin & $83(82.2 \%)$ & $17(16.8 \%)$ & $\mathrm{I}(\mathrm{I} .0 \%)$ & $69(76.7 \%)$ & $18(20.0 \%)$ & $3(3.3 \%)$ \\
\hline \multicolumn{7}{|l|}{ Non-hematologic toxic side effects } \\
\hline Increase in bilirubin & $84(83.2 \%)$ & $17(16.8 \%)$ & $0(0.0 \%)$ & $77(85.6 \%)$ & $13(14.4 \%)$ & $0(0.0 \%)$ \\
\hline Increase in transaminase & $61(60.4 \%)$ & $39(38.6 \%)$ & $\mathrm{I}(\mathrm{I} .0 \%)$ & $60(66.7 \%)$ & $30(33.3 \%)$ & $0(0.0 \%)$ \\
\hline Increase in alkaline phosphatase & $85(84.2 \%)$ & $16(15.8 \%)$ & $0(0.0 \%)$ & $76(84.4 \%)$ & $14(\mid 5.6 \%)$ & $0(0.0 \%)$ \\
\hline Increase in creatinine & $101(100 \%)$ & $0(0.0 \%)$ & $0(0.0 \%)$ & $88(97.8 \%)$ & $2(2.2 \%)$ & $0(0.0 \%)$ \\
\hline Nausea, vomiting & $27(26.7 \%)$ & $65(64.4 \%)$ & $9(8.9 \%)$ & $30(33.3 \%)$ & $5 \mathrm{I}(56.7 \%)$ & $9(10.0 \%)$ \\
\hline Oral mucositis & $66(65.3 \%)$ & $32(31.7 \%)$ & $3(3.0 \%)$ & $54(60.0 \%)$ & $35(38.9 \%)$ & $\mathrm{I}(\mathrm{I} .1 \%)$ \\
\hline Diarrhea & $66(65.3 \%)$ & $35(34.7 \%)$ & $0(0.0 \%)$ & $62(68.9 \%)$ & $27(30.0 \%)$ & $\mathrm{I}(\mathrm{I} .1 \%)$ \\
\hline Constipation & $78(77.2 \%)$ & $22(21.8 \%)$ & $\mathrm{I}(\mathrm{I} .0 \%)$ & $73(81.1 \%)$ & $17(18.9 \%)$ & $0(0.0 \%)$ \\
\hline Peripheral neuropathy & $57(56.4 \%)$ & $42(41.6 \%)$ & $2(2.0 \%)$ & $4 \mathrm{l}(45.6 \%)$ & $46(51.1 \%)$ & $3(3.3 \%)$ \\
\hline
\end{tabular}

Abbreviations: PNI, prognostic nutrition index; Low PNI group, PNI $\leq 47.77$; High PNI group, PNI>47.77; 
Table 3 The Relationship Between the PNI and the Toxic Side Effects of Concomitant Radiochemotherapy Case (Rate)

\begin{tabular}{|c|c|c|c|c|c|c|}
\hline \multirow{2}{*}{$\begin{array}{l}\text { Toxic Side-Effects of Concomitant } \\
\text { Radiochemotherapy }\end{array}$} & \multicolumn{3}{|c|}{ High PNI Group (47 Cases) } & \multicolumn{3}{|c|}{ Low PNI Group (46 Cases) } \\
\hline & Grade 0 & $\begin{array}{l}\text { Grade } \\
\mathrm{I}-2\end{array}$ & $\begin{array}{l}\text { Grade } \\
3-4\end{array}$ & Grade 0 & $\begin{array}{l}\text { Grade } \\
\mathrm{I}-2\end{array}$ & $\begin{array}{l}\text { Grade } \\
3-4\end{array}$ \\
\hline \multicolumn{7}{|l|}{ Hematologic toxic side effects } \\
\hline Leukocytosis & 18(38.3\%) & $22(46.8 \%)$ & $7(14.9 \%)$ & $16(34.8 \%)$ & $20(43.5 \%)$ & $10(21.7 \%)$ \\
\hline Decrease in platelet & $44(93.6 \%)$ & $3(6.4 \%)$ & $0(0.0 \%)$ & $42(91.3 \%)$ & $2(4.3 \%)$ & $2(4.3 \%)$ \\
\hline Decrease in hemoglobin & $38(80.9 \%)$ & $9(19.1 \%)$ & $0(0.0 \%)$ & $36(78.3 \%)$ & $8(17.4 \%)$ & $2(4.3 \%)$ \\
\hline \multicolumn{7}{|l|}{ Non- hematologic toxic side effects } \\
\hline Skin & $32(68.1 \%)$ & $15(31.9 \%)$ & $0(0.0 \%)$ & $27(58.7 \%)$ & $19(41.3 \%)$ & $0(0.0 \%)$ \\
\hline Esophagitis * & $40(85.1 \%)$ & $7(14.9 \%)$ & $0(0.0 \%)$ & $44(95.7 \%)$ & $2(4.3 \%)$ & $0(0.0 \%)$ \\
\hline Upper gastrointestinal symptoms * & $21(44.7 \%)$ & $25(53.2 \%)$ & $\mathrm{I}(2.1 \%)$ & $17(37.0 \%)$ & $27(58.7 \%)$ & $2(4.3 \%)$ \\
\hline Lower gastrointestinal symptoms * & $42(89.4 \%)$ & $5(10.6 \%)$ & $0(0.0 \%)$ & $4 I(89.1 \%)$ & $5(10.9 \%)$ & $0(0.0 \%)$ \\
\hline Fatigue and weakness & $34(72.3 \%)$ & $\mathrm{II}(23.4 \%)$ & $2(4.3 \%)$ & $32(69.6 \%)$ & $12(26.1 \%)$ & $2(4.3 \%)$ \\
\hline
\end{tabular}

Notes: *Esophagitis: pain behind the sternum, burning sensation, changes in eating or swallowing. Upper gastrointestinal symptoms: anorexia, nausea, vomiting, and upper abdomen pain. Lower gastrointestinal symptoms: abdominal pain, bloating, diarrhea, constipation.

Abbreviations: PNI, prognostic nutrition index; Low PNI group, PNI $\leq 47.77$; High PNI group, PNI>47.77.

$89.5 \%, 69.8 \%$, and $57.1 \%$, respectively. The $1-, 2$-, and 3-year survival rates of high PNI group and low PNI group were $92.1 \%$ vs $86.7 \%(P=0.210), 77.9 \%$ vs $60.6 \%$ $(P=0.007)$ and $66.2 \%$ vs $46.7 \%(P=0.003)$, respectively, and the 5 -year survival rate was $38.2 \%$ for the whole group. The 5-year survival rate in the high PNI group and the low PNI group was $49.8 \%$ and $24.2 \%(\mathrm{P}=0.001)$. The variables were introduced into the Cox regression models for the analysis of factors affecting survival. The single factor analysis showed that nerve or vascular infiltration $(p=0.030)$, TNM stage $(p=0.000)$, PNI ( $p=0.001)$, and chemotherapy cycles $(p=0.045)$ were strongly associated with survival prognosis. The multifactor analysis showed that PNI $(\mathrm{p}=0.001)$ and TNM stage $(\mathrm{p}=0.000)$ were independent risk factors for the overall survival time after surgery in patients with gastric cancer (Table 4).

\section{Discussion}

The nutritional status in patients with cancer can affect the immune response and healing of the surgical incision, which, in turn, affect the prognosis. ${ }^{8,9}$ Severely malnourished patients with cancer have significantly higher mortality, ${ }^{10}$ while for patients with gastric cancer, because of the tumor itself, malnutrition is more likely to occur. Several assessment tools are currently available for the assessment of nutritional status: Nutritional Risk
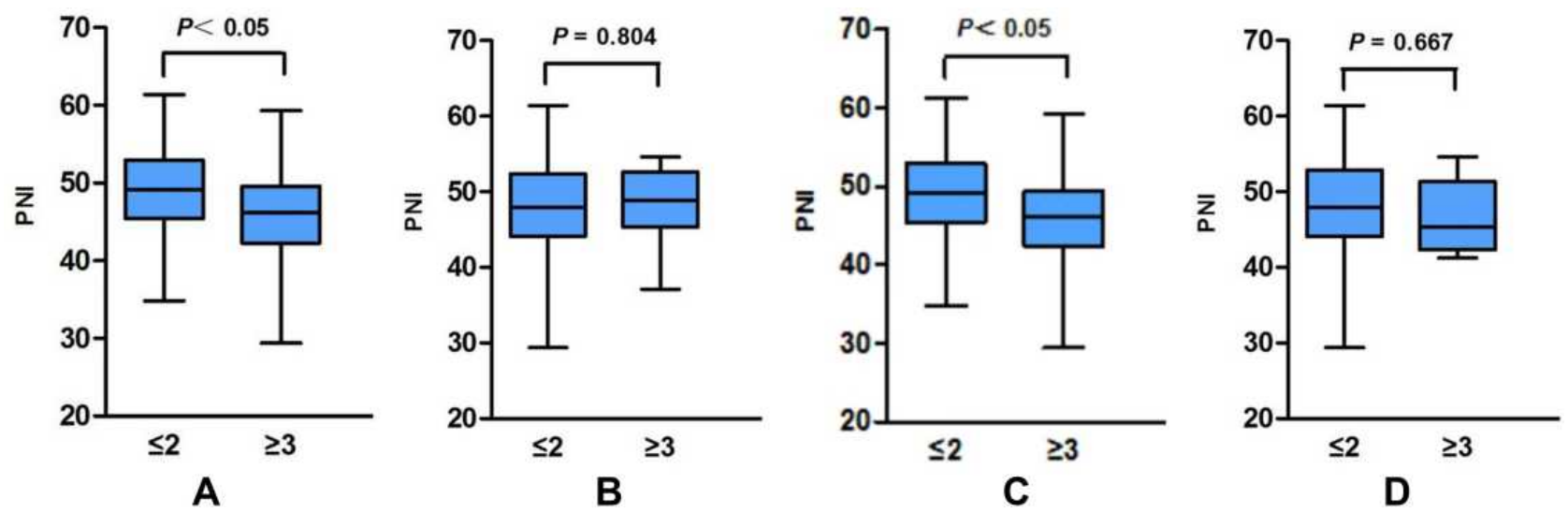

Figure 2 PNI and the grade 0-2, 3-4 haematological and non-haematological toxic side-effects of radiochemotherapy. (A) Grades of the haematological toxic side-effects of adjuvant chemotherapy. (B) Grades of the non-haematological toxic side-effects of adjuvant chemotherapy. (C) Grades of the haematological toxic side-effects of the concomitant radiochemotherapy. (D) Grades of the non-haematological toxic side-effects of the concomitant radiochemotherapy. 


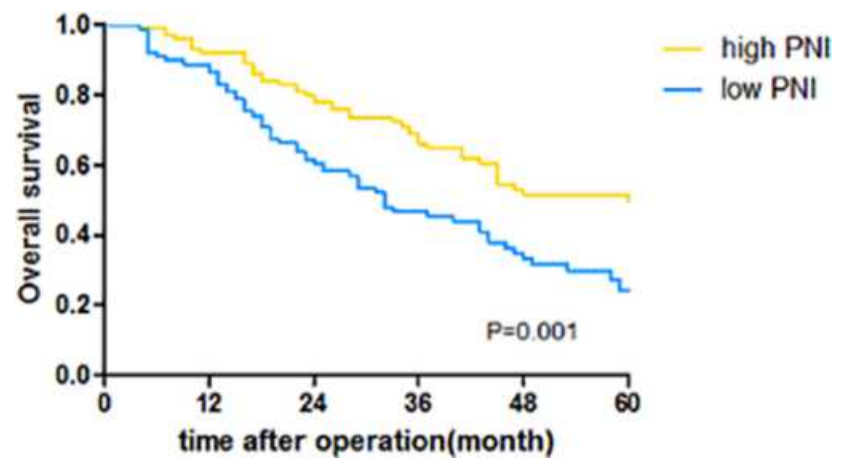

Figure 3 Curves of the overall survival time in patients in the high PNI group and low PNI group with gastric cancer after surgery.

Screening (NRS2002), Controlling Nutritional Status (CONUT) score, and the Subjective General Nutrition Assessment (SGA). It has been reported that a high CONUT score could increase the incidence of postoperative infection and was an independent risk factor for the survival time, ${ }^{11}$ and a high NRS2002 score was associated with prolonged postoperative hospitalization and postoperative complications; ${ }^{12}$ however, these assessment tools are cumbersome to use and have little clinical utility. It has also been reported that immunity is strongly associated with the prognosis of patients with malignant tumors. $^{13}$ In recent years, a number of clinical indices incorporating the nutritional and inflammatory parameters have emerged to predict the risk of surgery and the outcome of a tumor. Among them, the PNI, which is composed of the level of serum albumin and total peripheral blood lymphocyte count, reflects the nutritional status and immunity. When prospectively applied to patients undergoing gastrointestinal surgery, this index provides an accurate estimate of the risk of surgery. ${ }^{5}$ In recent years, studies have shown the predictive value of the PNI in the prognosis of gastric cancer, colorectal cancer, pancreatic cancer, and esophageal cancer. ${ }^{14-17}$ The results of this study showed that the 1 -, 2 -, and 3-year survival rates of the high PNI group were higher than those of the low PNI group, which were similar to the results reported in the literature. ${ }^{18,19}$ The results of this study suggested that the 2 -, and 3-year survival rates of the high PNI group were significantly higher than those of the low PNI group. Some relevant domestic studies showed that among patients with gastric cancer, the 5-year survival rate in the high PNI group ranged from $34.2 \%$ to $54.1 \%$, and the 5-year survival rate in the low PNI group ranged from $8.9 \%$ to $37.7 \% .^{20-22}$ The results of the present study showed that the 5-year survival rate was significantly higher in the high PNI group than in the low PNI group (49.8\% vs $24.2 \% \mathrm{p}=0.001$ ), which is consistent with the results of previous studies in the literature. However, it should be noted that there was no clear definition of the PNI cutoff in those studies, and most of the studies were based on the best cutoff for predicting the 5 -year survival as the cutoff point. Furthermore, the overall nutritional status of the patients included in the analysis might vary from one study to another, resulting in a different survival prognosis. The overall 5-year survival rate in the present study remained low, the possible reasons for which might be related to the following factors: there were relatively more patients in stage III, accounting for $70.2 \%$ of the sample; and some patients failed to complete the full cycle of chemotherapy for their own reasons or because of the toxic side effects of chemotherapy. The results of the present study also showed that the

Table 4 Single-Factor and Multifactor Analysis of the Overall Survival Time in Patients After the Gastric Cancer Surgery

\begin{tabular}{|c|c|c|c|c|}
\hline \multirow[t]{2}{*}{ Variable } & \multicolumn{2}{|c|}{ Single-Factor } & \multicolumn{2}{|c|}{ Multifactor } \\
\hline & HR $(95 \% \mathrm{Cl})$ & $\mathbf{P}$ & HR $(95 \% \mathrm{Cl})$ & $\mathbf{P}$ \\
\hline Gender (Male/Female) & $1.32(0.87-2.02)$ & 0.196 & & \\
\hline Age $(\geq 65 /<65)$ & $1.42(0.93-2.15)$ & 0.105 & & \\
\hline Degree of tumor differentiation (intermediate to high-differentiated/low- differentiated) & $\mathrm{I} .45(0.84-2.50)$ & 0.184 & & \\
\hline $\mathrm{BMI}(\geq \mid 8.5 /<18.5)$ & $1.23(0.77-1.95)$ & 0.384 & & \\
\hline Nerve or vascular invasion (With invasion/Without invasion) & $1.55(1.04-2.30)$ & 0.030 & $1.40(0.94-2.09)$ & 0.094 \\
\hline TNM stage (I-II/III) & $3.91(2.25-6.78)$ & 0.000 & $3.78(2.16-6.59)$ & 0.000 \\
\hline Radiotherapy (With radiotherapy/Without radiotherapy) & $1.46(0.97-2.13)$ & 0.052 & & \\
\hline Cycles of chemotherapy ( $3-5$ cyces/6-8 cycles) & $1.51(1.01-2.26)$ & 0.045 & $1.39(0.93-2.10)$ & 0.111 \\
\hline PNI (High PNI group/Low PNI group) & $1.89(1.29-2.77)$ & 0.001 & $1.88(1.28-2.78)$ & 0.001 \\
\hline
\end{tabular}

Abbreviations: PNI, prognostic nutrition index; HR, hazard ratio; BMI, body mass index; TNM, tumor-node-metastasis. 
PNI was an independent prognostic factor for the overall postoperative survival time in patients with gastric cancer, which is consistent with the findings of previous studies. $^{23,24}$

As one of the main treatments for patients with stage Ib-III gastric cancer, radiochemotherapy can improve the prognosis and prolong the survival time; however, it can also cause different degrees of toxic side effects. Severe side effects may cause anxiety, loss of confidence in the treatment, or intolerance leading to treatment discontinuation, affecting the survival prognosis. Therefore, the ability to predict the toxic side effects of radiochemotherapy in patients with cancer and to implement early interventions to reduce the occurrence of toxic side effects is crucial. Studies on the toxic side effects of chemotherapy have shown that female gender, low BMI, and hypoalbuminemia are independent prognostic factors for the hematologic toxic side effects of grade 3-4 chemotherapy. ${ }^{25}$ Guo et al reported that patients with gastric cancer with a high preoperative PNI had fewer and less severe adverse effects of chemotherapy than those with a low PNI. ${ }^{20}$ A study of patients receiving neoadjuvant chemotherapy for gastric cancer showed that post-chemotherapy anemia and lymphopenia were significantly associated with a lower prechemotherapy PNI. ${ }^{18}$ There are few studies on the association between nutrition and the side effects of radiotherapy; however, some researchers believe that a low PNI and a high CONUT score are closely connected with severe side effects of radiotherapy in patients with head and neck tumors. ${ }^{26}$ In patients receiving adjuvant radiochemotherapy for esophageal cancer, patients with a low PNI were more likely to have hematologic toxic side effects of radiotherapy above grade 3 , and the correlation coefficient between PNI and toxic side effects was higher than that between lymphocyte count and serum albumin levels. ${ }^{6}$ However, there are few studies on the relationship between the PNI and chemotherapeutic side effects in gastric cancer, and no study concerning the correlation between the PNI and side effects of radiotherapy has been reported. The results of the present study showed that patients with a low PNI were more likely to experience hematologic toxic side effects of radiochemotherapy above grade 3 . Therefore, preoperative nutritional monitoring in patients with gastric cancer and appropriate supportive therapy for patients with a low PNI might help to improve the nutritional status and immunity, which might help them to successfully complete the adjuvant radiochemotherapy and improve their survival prognosis.
Because of the small sample in the present study, a prospective study with a larger sample size is needed to provide a more accurate assessment of the relationship between nutritional status and adjuvant radiochemotherapy in patients with gastric cancer.

In summary, the results of the present study suggested that the preoperative PNI could predict the severity of hematologic side effects of adjuvant chemotherapy/radiotherapy in patients with gastric cancer, and that the group with a low PNI was more likely to have severe hematologic side effects, which was one of the important factors affecting the prognosis in patients with gastric cancer. Further clinical study is necessary to verify this conclusion.

\section{Ethics Approval and Consent to Participate}

This study was conducted in accordance with the Declaration of Helsinki. This study was conducted with approval from the Ethics Committee of Affiliated Hospital of Guizhou Medical University. A written informed consent was obtained from all participants.

\section{Consent for Publication}

Consent for publication was obtained from every individual whose data are included in this manuscript.

\section{Funding}

This work was supported by Guizhou science and Technology Department (E2009-29).

\section{Disclosure}

All authors declare that the work is original and has not been submitted or published elsewhere. None of the authors have any financial disclosure or conflicts of interest.

\section{References}

1. National Health Commission. Guidelines for the Diagnosis and Treatment of Gastric Cancer (2018 Edition). Chin J Dig Dis Imaging. 2019;9(003):118-144.

2. Association J G C. Japanese gastric cancer treatment guidelines 2018 (5th edition). Gastric Cancer. 2020;(Suppl 1):1-21.

3. NCCN Clinical Practice Guidelines in Oncology (Gastric Cancer) -V.2 2020. Available from: www.nccn.org. Accessed March 26, 2021.

4. Shi HP, Li SY, Wang KH, et al. Guidelines for nutritional treatment of gastric cancer patients. Clin Educ General Pract. 2015;13(5):488-491 +514 .

5. Onodera T, Goseki N, Kosaki G. Prognostic nutritional index in gastrointestinal surgery of malnourished cancer patients. Nippon Geka Gakkai Zasshi. 1984;85(9):1001-1005. 
6. Matsumoto Y, Zhou Q, Kamimura K, Moriyama M, Saijo Y. The prognostic nutrition index predicts the development of hematological toxicities in and the prognosis of esophageal cancer patients treated with cisplatin plus 5-fluorouracil chemotherapy. Nutr Cancer. 2018;70:447-452. doi:10.1080/01635581.2018.1445765

7. Common Terminology Criteria for Adverse Events v 3.0. Int J Clin Oncol. 2004;9.

8. Morgan TM, Tang D, Stratton KL, et al. Preoperative nutritional status is an important predictor of survival in patients undergoing surgery for renal cell carcinoma. Eur Urol. 2011;59(6):923-928. doi:10.1016/j.eururo.2011.01.034

9. Lee H, Cho YS, Jung S, Kim H. Effect of nutritional risk at admission on the length of hospital stay and mortality in gastrointestinal cancer patients. Clin Nutr Res. 2013;2(1):12-18. doi:10.7762/cnr.2013.2.1.12

10. La Torre M, Ziparo V, Nigri G, Cavallini M, Balducci G, Ramacciato G. Malnutrition and pancreatic surgery: prevalence and outcomes. J Surg Oncol. 2013;107(7):702-708. doi:10.1002/jso.23304

11. Satoshi S, Shingo K, Masashi Y, et al. Controlling Nutritional Status (CONUT) score predicts outcomes of curative resection for gastric cancer in the elderly. World J Surg. 2019;43:1076-1084. doi:10.1007/ s00268-018-04889-6

12. Guo W, Ou G, Li X, Huang J, Liu J, Wei H. Screening of the nutritional risk of patients with gastric carcinoma before operation by NRS 2002 and its relationship with postoperative results. J Gastroenterol Hepatol. 2010;25(4):800-803. doi:10.1111/j.1440-1746.2009.06198.x

13. Saito H, Shimizu S, Kono Y, et al. Score of the preoperative absolute number of lymphocytes, monocytes, and neutrophils as a prognostic indicator for patients with gastric cancer. Surg Today. 2019;49:850-858. doi:10.1007/s00595-019-01817-6

14. Nozoe T, Kohno M, Iguchi T, et al. The prognostic nutritional index can be a prognostic indicator in colorectal carcinoma. Surg Today. 2012;42:532-535. doi:10.1007/s00595-011-0061-0

15. Kanda M, Fujii T, Kodera Y, Nagai S, Takeda S, Nakao NA. Nutritional predictors of postoperative outcome in pancreatic cancer. Br J Surg. 2011;98(2):268-274. doi:10.1002/bjs.7305

16. Nozoe T, Kimura Y, Ishida M, Saeki H, Korenaga D, Sugimachi K. Correlation of pre-operative nutritional condition with post-operative complications in surgical treatment for oesophageal carcinoma. Eur J Surg Oncol. 2002;28(4):396-400. doi:10.1053/ejso.2002.1257
17. Sakurai K, Tamura T, Toyokawa T, et al. Low preoperative prognostic nutritional index predicts poor survival post-gastrectomy in elderly patients with gastric cancer. Ann Surg Oncol. 2016;23:3669-3676. doi:10.1245/s10434-016-5272-6

18. Sun J, Wang D, Mei Y, et al. Value of the prognostic nutritional index in advanced gastric cancer treated with preoperative chemotherapy. J Surg Res. 2016;209:37-44. doi:10.1016/j.jss.2016.09.050

19. Tadahiro N, Mizuki N, Takashi M, et al. Prognostic nutritional index: a tool to predict the biological aggressiveness of gastric carcinoma. Surg Today. 2010;40:440-443. doi:10.1007/s00595-009-4065-y

20. Guo JY, Fang LJ, Guo JY. Correlation analysis between prognostic nutritional index and clinical features and prognosis of resectable gastric cancer. Chinese J Clin Oncol. 2015;42(2):100-104.

21. Jiang N, Deng JY, Ding XW, et al. Prognostic nutritional index predicts postoperative complications and long-term outcomes of gastric cancer. World J Gastroenterol. 2014;20:10537-10544. doi:10.3748/wjg.v20.i30.10537

22. Song SB, Liu HG, Xue YW. Clinical significance of prognostic nutritional index in patients with advanced gastric cancer. Chin $J$ Gastrointestinal Surg. 2018;2:180-184.

23. Kanda M, Mizuno A, Tanaka C, et al. Nutritional predictors for postoperative short-term and long-term outcomes of patients with gastric cancer. Medicine (Baltimore). 2016;95:e3781. doi:10.1097/ MD.0000000000003781

24. Sakurai K, Ohira M, Tamura T, et al. Predictive potential of preoperative nutritional status in long-term outcome projections for patients with gastric cancer. Ann Surg Oncol. 2016;23:525-533. doi:10.1245/s10434-015-4814-7

25. Seo SH, Kim SE, Kang YK, et al. Association of nutritional status-related indices and chemotherapy-induced adverse events in gastric cancer patients. BMC Cancer. 2016;16:900. doi:10.1186/ s12885-016-2934-5

26. Takeyuki K, Koji S, Seiichi S, et al. Pre-therapeutic nutritional assessment for predicting severe adverse events in patients with head and neck cancer treated by radiotherapy. Clin Nutr. 2017;36:1681-1685.

\section{Publish your work in this journal}

Cancer Management and Research is an international, peer-reviewed open access journal focusing on cancer research and the optimal use of preventative and integrated treatment interventions to achieve improved outcomes, enhanced survival and quality of life for the cancer patient.
The manuscript management system is completely online and includes a very quick and fair peer-review system, which is all easy to use. Visit http://www.dovepress.com/testimonials.php to read real quotes from published authors. 